ISSN: 2602-8085

\title{
Evaluación del rendimiento de la modulación Binary Offset Carrier (BOC) versus la señal Civil GPS/L1
}

Performance evaluation of the Binary Offset (BOC) Carrier modulation versus GPS/L1 Civil Signal

Andrea Magali Llerena Pintag. ${ }^{1}$, Mónica Andrea Zabala Haro. ${ }^{2}$, Mayra Alejandra Pacheco Cunduri. ${ }^{3}$, Byron Andres Casignia Vasconez. ${ }^{4}$ \& Mayra Alejandra Oñate Andino. ${ }^{5}$

Recibido: 19-04-2019 / Revisado: 24-05-209 /Aceptado: 25-06-2019/ Publicado: 05-07-2019

\begin{abstract}
DOI: https://doi.org/10.33262/cienciadigital.v3i3.626

This article is a comparison of evaluation of the performance of GPS L1 satellite signals modulation techniques, Binary Offset Carrier (BOC) vs. Binary Phase Shift Keying (BPSK). The state of the art of the GPS positioning system since its launch to the present day. The development of the simulation is carried out using the mathematical software Matlab and parameters were analyzed for the generation and conditioning of the signal of GPS L1 civil satellite, to be encoded using the Convolutional encoder $(2,1,3)$ and modulated with BOC before be sent to the transmission channel. Two fading channels type Rayleigh and Rician were analyzed in the transmission stage. Stage results are detailed charts and tables of data obtained by simulation to evaluate the performance of the channel as a function of BER and SNR parameters.
\end{abstract}

Keywords: Global Positioning System, GPS, Modulation, GNSS Signals, Fading Channels.

\section{Resumen}

En este artículo se realiza una comparación de evaluación del rendimiento de las técnicas de modulación de señales satelitales de GPS L1, Binary Offset Carrier (BOC) vs. Binary

${ }^{1}$ Escuela Superior Politécnica de Chimborazo, Ingeniera en Electrónica, Control y Redes Industriales, Chimborazo, Ecuador. magillerena@gmail.com

${ }^{2}$ Escuela Superior Politécnica de Chimborazo, Facultad de Informática y Electrónica, Escuela de Ingeniería Electrónica en Telecomunicaciones y Redes, Chimborazo, Ecuador, m_zabala@espoch.edu.ec

${ }^{3}$ Escuela Superior Politécnica de Chimborazo, Facultad de Informática y Electrónica, Escuela de Ingeniería Electrónica en Telecomunicaciones y Redes, Chimborazo, Ecuador

${ }^{4}$ Escuela Superior Politécnica de Chimborazo, Carrera de Electrónica y Telecomunicaciones, Chimborazo, Ecuador, bcasignia@unach.edu.ec

${ }^{5}$ Escuela Superior Politécnica de Chimborazo, Facultad de Informática y Electrónica, Escuela de Ingeniería Electrónica en Telecomunicaciones y Redes, Chimborazo, Ecuador, monate @espoch.edu.ec 
ISSN: 2602-8085

Phase Shift Keying (BPSK). Se realiza un análisis del estado del arte del sistema de posicionamiento GPS desde su lanzamiento hasta la actualidad. El desarrollo de la simulación se realiza utilizando el software matemático Matlab y se analizaron parámetros para la generación y acondicionamiento de la señal satelital civil de GPS L1, para ser codificada mediante el codificador convolucional $(2,1,3)$ y modulada con BOC antes de ser enviada al canal de transmisión. En la etapa de trasmisión se analizaron dos canales de desvanecimiento tipo Rayleigh y Rician. En su etapa de resultados se detallan los gráficos y tablas de los datos obtenidos por la simulación para evaluar el rendimiento del canal en función de los parámetros BER y SNR.

Palabras Claves: Sistema de Posicionamiento Global, GPS, Modulación, Señales GNSS Canales De Desvanecimiento.

\section{Introducción}

El sistema de posicionamiento global (GPS) que durante más de 2 décadas desde su aparición, es el sistema más utilizado y conocido tanto para uso civil y como militar. En la actualidad son innumerables la cantidad de aplicaciones que se han desarrollado, con el paso de los años; países potencias mundiales como Rusia, Europa y China, han ido desarrollando nuevos sistemas de posicionamiento los mismos que han tenido inconvenientes por no estar en completa operatividad, ésta es la razón por la que la mayoría de personas siguen usando y están muy familiarizadas con el término GPS y no con los demás sistemas GNSS como son Glonass, Galileo, BeiDou. (Valadez D., 2015).

GPS ofrece los servicios Standard Positioning Service (SPS) y Precise Positioning Service (PPS). El primero se encuentra a disposición de la sociedad civil, en la frecuencia L1 $=1575.42$ $\mathrm{MHz}$ y el segundo de uso restringido solamente de uso militar opera la frecuencia L2 $=1227.60$ $\mathrm{MHz}$, ambos servicios utilizan modulación Binary Phase Shift Key (BPSK), (HofmannWellenhof, Lichtenegger, \& Wasle, 2008, pág. 315). Para proporcionar interoperabilidad de GPS y los nuevos sistemas de navegación se han propuesto nuevos modelos de modulación entre los cuales se encuentra la modulación Binary Offset Carrier (BOC).

\section{Desarrollo}

\section{Modulación BPSK}

BPSK se define por una portadora de Radio Frecuencia que se transmite desplazada $180^{\circ}$, dependiendo si el bit que se transmite corresponde a un 1 lógico o un 0 lógico (Reyes, 2012). La fórmula 1 corresponde a la señal BPSK utilizada para GPS (Serna, 2015).

$$
S^{k}=A_{c}(C(t) X O R D(t)) \cos \left(2 \pi f_{l} t+\varnothing\right)
$$

donde: 
- $S^{\mathrm{k}}: \mathrm{k}^{\text {th }}$ Satélite GPS

- $\mathrm{A}_{\mathrm{c}}$ : Amplitud del código C/A

- $\mathrm{C}(\mathrm{t}): \pm 1$ indica la fase del código $\mathrm{C} / \mathrm{A}$

- $\mathrm{D}(\mathrm{t}): \pm 1$ indica la fase de la señal de datos

- $\mathrm{f}_{1}$ : Frecuencia $\mathrm{L} 1=1575.42 \mathrm{MHz}$

- $\varnothing$ : Fase inicial

\section{Técnica DSSS}

El Espectro Ensanchado por Secuencia Directa o DSSS por sus siglas en inglés, es una técnica de Spread Spectrum aplicable a cualquier esquema de modulación digital como la BPSK. Se denomina espectro ensanchado, debido a que se aumenta el ancho de banda que ocupa la señal luego de ser modulada por la elevada velocidad de la onda PRN, a esto se lo conoce como chipping. El ancho de banda de la señal de espectro ensanchado es proporcional a la razón de chipping.

Existen sistemas de navegación como el sistema GPS que utilizan esta técnica, debido a sus beneficios ante la presencia de interferencias y efectos de multicamino, la inversión constante del código PRN que permite una mejor precisión en el receptor, además del uso de varias y diversas secuencias PRN permite la transmisión simultánea de diversos satélites en la misma frecuencia.

Los sistemas que usan la técnica DSSS cumplen características como: El AB (ancho de banda) de la señal $m(t)$ que corresponde al mensaje de navegación es menor que el AB de la señal $s(t)$ que corresponde a la señal enviada. La fórmula 2 corresponde a la señal DSSS con corrimiento de fase binaria BPSK. (Armijo, 2017).

$s(t)=R e *\left[A_{c} m(t) * c(t) * e^{j w_{c} t}\right]$

Dónde:

- $A_{c} m(t)$ : Envolvente Compleja

- $c(t)$ : Señal Ensanchadora

El ancho de banda de la señal $s(t)$ se forma por una señal ensanchadora $c(t)$ y esta señal debe ser conocida por el receptor para la recuperación de los bits transmitidos. La señal ensanchadora $c(t)$ o también llamada PRN o código PRN es similar a la forma de onda de la señal de datos, lo que las diferencia es la razón de símbolo mucho mayor, su forma de onda es periódica y finita. 
Figura. 1. Señal modulada DSSS

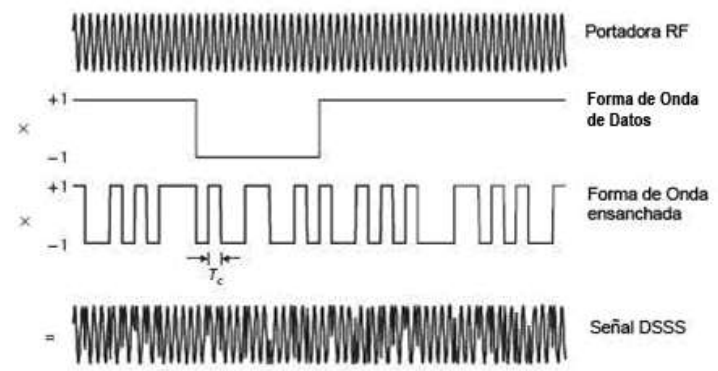

Modulación BOC

La modulación BOC surge por la necesidad de añadir una nueva señal DSSS (Reyes, 2012) dentro de las bandas de frecuencias que están en funcionamiento, además de mejorar las propiedades tradicionales de las señales GNSS como resistencia al multitrayecto, interferencias de todo tipo de ruido y básicamente para una mejor compartición espectral del ancho de banda con las señales existentes o futuras; es decir describe una clase de modulación de espectro ensanchado introducidas recientemente para la nueva generación de sistemas satelitales. La nueva generación de satélites GPS incluirá este tipo de modulación.

La modulación BOC, se define, como el producto en el dominio del tiempo de una señal portadora sinusoidal, subportadora, código de expansión PRN y la señal de datos. Tanto la frecuencia de subportadora $f_{s}$ como la tasa del código PRN son dos parámetros de diseño independientes en la modulación. Estos dos componentes son los que proporcionan independencia para concentrar la potencia de la señal dentro de cualquier lugar de la banda asignada y reducir la interferencia con otras señales.

Los vehículos espaciales tienen un reloj atómico con una frecuencia nominal referencial de $1.023 \mathrm{MHz}$, de este se generan las componentes de la señal de navegación. Para la señal BOC sus componentes se eligen como múltiplos de fo.

$$
\begin{aligned}
& f_{s}=m * f_{o}=\frac{1}{2 T_{s}} \\
& f_{c}=n * f_{o}=\frac{1}{n T_{s}}=\frac{2}{n} f_{s}
\end{aligned}
$$

Dónde:

- m: Define la frecuencia de subportadora

- n: Define la tasa de código PRN

$$
\mathrm{r}=2 \frac{\mathrm{f}_{\mathrm{s}}}{\mathrm{f}_{\mathrm{c}}}=2 \frac{\mathrm{m}}{\mathrm{n}}
$$

La razón $r$ es el número de períodos de la subportadora durante un código de chip. Se denomina modulación BOC $(\mathrm{m}, \mathrm{n})$, donde $m$ y $n$ siempre serán números enteros naturales. Como la 
modulación BOC es una modulación de onda subportadora cuadrada; donde una señal $s(t)$ se multiplica por una subportadora rectangular de frecuencia $f s c$, que divide el espectro de la señal en dos partes, por lo tanto las fórmulas 5 y 6 corresponden a la señal BOC. (Usha, SuryaPrabha, \& Dr.V.B.S.Srilatha-Indira, 2012).

$$
\begin{aligned}
& \mathrm{S}_{\mathrm{boc}}(\mathrm{t})=\mathrm{s}(\mathrm{t}) * \operatorname{sign}\left(\sin \left(2 \pi \mathrm{f}_{\mathrm{sc}} \mathrm{t}\right)\right) \\
& \mathrm{S}_{\mathrm{boc}}(\mathrm{t})=\mathrm{s}(\mathrm{t}) * \operatorname{sign}\left(\cos \left(2 \pi \mathrm{f}_{\mathrm{sc}} \mathrm{t}\right)\right)
\end{aligned}
$$

\section{Características de la señal BOC}

Una de las características que proporciona la presencia de la subportadora es la función de autocorrelación (ACF) de múltiples picos que divide la densidad espectral de potencia (PSD) en dos lóbulos simétricos separados de la frecuencia central de la señal (Betz, 1999). La ACF y PSD de BPSK y BOC $(1,1)$ se muestran en la figura 3 , en el caso de BOC $(1,1)$ la pendiente es más pronunciada en comparación con el ACF de BPSK, lo que proporciona una mayor capacidad de recuperación de datos frente a efectos de multicamino. Sin embargo, el ACF de las señales BOC tiene múltiples picos; uno primario y varios secundarios que pueden conducir a un seguimiento ambiguo. El número de picos secundarios $N s p$ en la señal modulada BOC se obtiene con la fórmula 7.

$$
\mathrm{N}_{\mathrm{sp}}= \begin{cases}4\left(\frac{\mathrm{m}}{\mathrm{n}}\right)-2 & \text { BOCs } \\ 4\left(\frac{\mathrm{m}}{\mathrm{n}}\right) & \text { BOCc }\end{cases}
$$

La figura 2, muestra el espectro de potencia simétrico con dos lóbulos principales desplazados de la frecuencia principal permite procesar señales BPSK y BOC simultáneamente con mínimas interferencias.

Figura 2. Función de ACF y PSD para las señales BPSK y BOC [7]
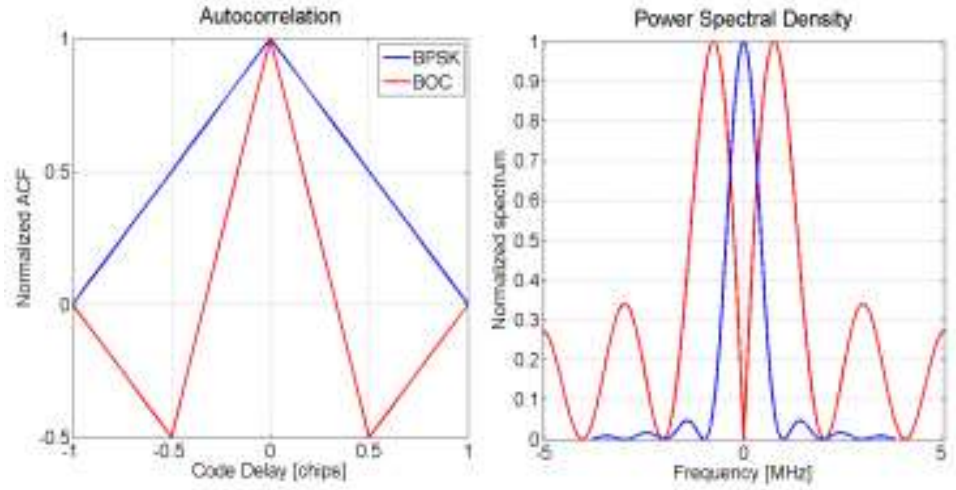
ISSN: 2602-8085

\section{Espectro de la Señal Civil GPS L1 y Galileo E1}

Los sistemas GPS y GALILEO emiten señal L1 y E1 respectivamente en frecuencias que se sobreponen para que ambos sistemas sean compatibles e interoperables. El uso combinado de ambos sistemas permite mejorar la precisión, integridad de la señal, disponibilidad y fiabilidad. La interoperabilidad se consigue mediante un solape parcial de frecuencias y/o diferentes secuencias de código. (Julien, Future GNSS Signals, 2014)

Figura 3. Señales Civiles de GPS y GALILEO - L1/E1

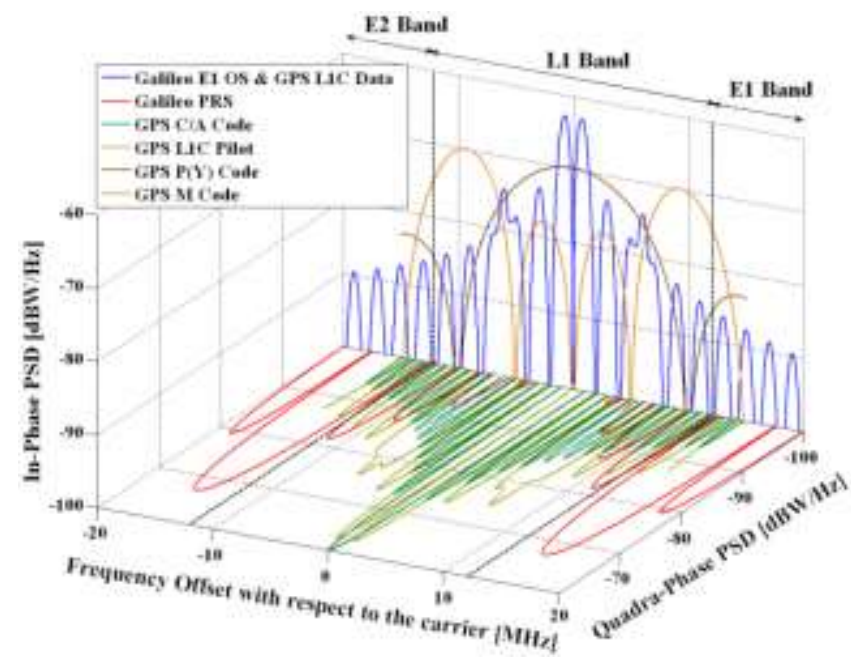

\section{Metodología}

La metodología a seguir se plasma en el diagrama de bloques tomado de (Armijo, 2017) y presentado en la Figura 4.

Figura 4. Diagrama de Bloques Generación, Tx y Rx de Señal GPS

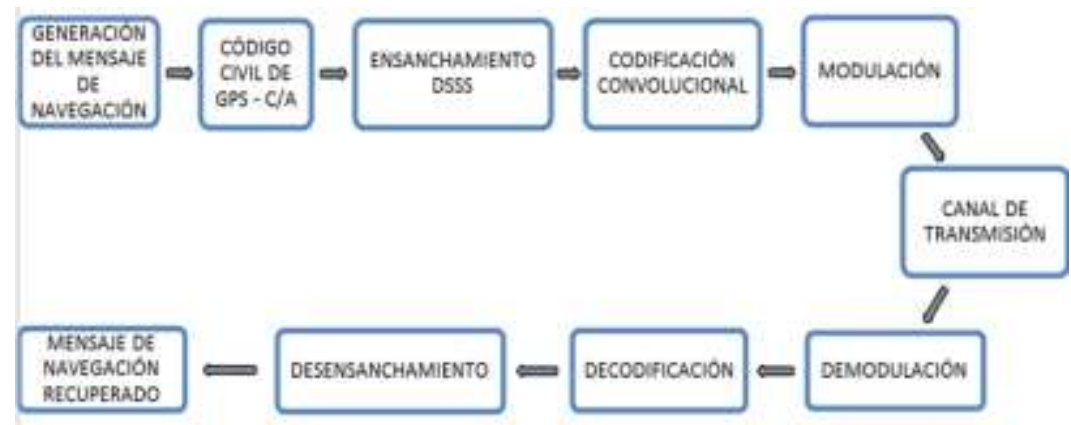

Las etapas del diagrama de bloques, desde la generación de la señal portador, generación del mensaje de navegación, formación del código Coarse Adquisition (C/A), la técnica de ensanchamiento de la señal y la codificación se detalla ampliamente en (Armijo, 2017). 
Para el proceso de demodulación a la señal recibida a la salida del canal de transmisión se multiplica por una réplica de la señal portadora cosenoidal. Las etapas de decodificación y desenchanchamiento, así como el proceso de recuperación del mensaje de navegación (NAV) se detalla ampliamente en (Armijo, 2017). Para realizar la comparación entre las modulaciones BPSK y BOC, se toman los valores de relación señal a ruido SNR para evaluar el canal de transmisión considerados en (Armijo, 2017). Los valores SNR corresponden a $0 \mathrm{~dB}, 2 \mathrm{~dB}, 4 \mathrm{~dB}$, $10 \mathrm{~dB}, 40 \mathrm{~dB}$.

Tabla 1 Generación de Señal GPS L1, Técnica de DSSS, canal y codificación

\begin{tabular}{ll}
\hline Concepto & Valor \\
\hline Número de Satélites GPS: & 1 \\
Frecuencia Fundamental: & $\mathrm{L} 1=1575.42 \mathrm{Mhz}$ \\
Mensaje de Navegación NAV & $50 \mathrm{~Hz}$ \\
Frecuencia del código C/A: & $1.023 \mathrm{Mhz}$ \\
Código Pseudorandómico - PRN & $1.023 \mathrm{chips}$ \\
Bits del Mensaje de Navegación & $1500 \mathrm{bits}$ \\
Codificación Convolucional $(2,1,3)$ & $\mathrm{k}=1, \mathrm{n}=2, \mathrm{~m}=3$. Razón $=1 / 2$ \\
Velocidad del Receptor & $0 \mathrm{~m} / \mathrm{s}, 20 \mathrm{~m} / \mathrm{s}, 60 \mathrm{~m} / \mathrm{s}$, y $100 \mathrm{~m} / \mathrm{s}$ \\
Relación Señal a Ruido & $0 \mathrm{~dB}, 2 \mathrm{~dB}, 4 \mathrm{~dB}, 10 \mathrm{~dB}$ y $40 \mathrm{~dB}$ \\
\hline
\end{tabular}

Fuente: Andrea Llerena, 2019

\section{Espectro de la señal GPS L1}

El análisis del espectro del mensaje de navegación en $\mathrm{dB}$, al que se le ha aplicado la modulación BPSK se puede observar en la Figura 5, mientras que en la Figura 6 se observa el mensaje de navegación aplicado la modulación BOC. El mensaje de navegación ensanchado, se logra con la suma en módulo 2 del código pseudorandómico C/A con los bits de información del mensaje.

Figura 5. Espectro del Mensaje de Navegación con Modulación BPSK

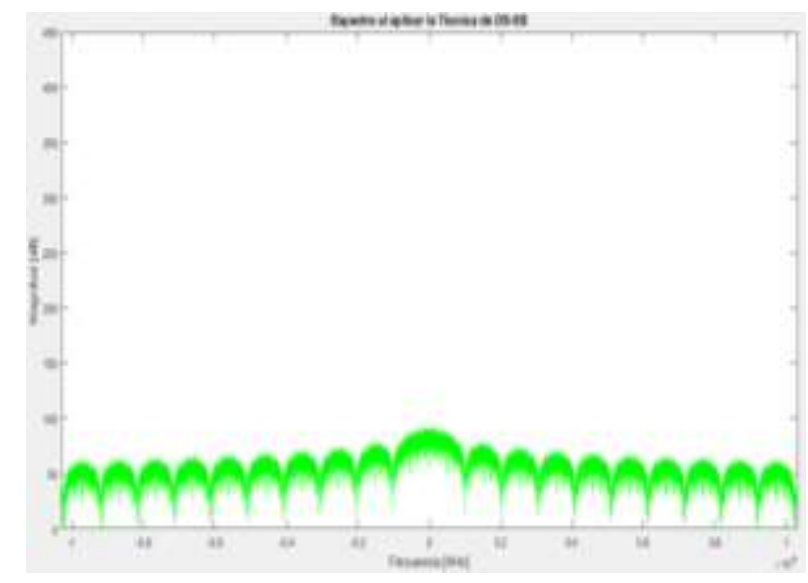


Figura 6. Espectro de Mensaje de Navegación aplicando Modulación BOC

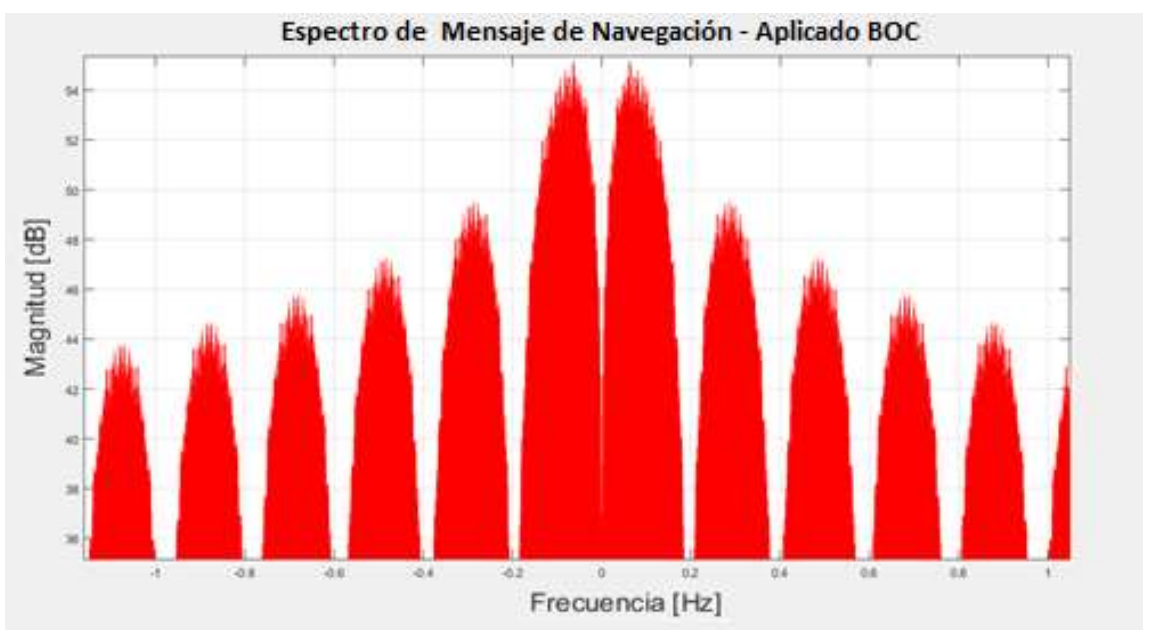

Como se observa, el ancho de banda ocupado por el espectro al que se le aplicó la modulación BPSK es de 2 Mhz en su único lóbulo principal, mientras que el ancho de banda ocupado por el espectro aplicado modulación BOC ocupa los mismos $2 \mathrm{Mhz}$ en sus dos lóbulos principales simétricos debido a la onda subportadora cuadrada característico de la modulación BOC.

\section{Canal de transmisión}

\section{Evaluación de la Modulación BPSK y BOC en canal de Rayleigh}

En las Tabla 2 y 3, se muestran los errores producidos dentro del canal con desvanecimiento tipo Rayleigh con y sin codificación Convolucional $(2,1,3)$, considerando las modulaciones BPSK y BOC, respectivamente. Además, se considera la variación de la velocidad de recepción y niveles SNR.

Tabla 2 BPSK con y sin codificación convolucional $(2,1,3)$

\begin{tabular}{llllllllll}
\hline $\mathrm{SNR}$ & $0 \mathrm{~m} / \mathrm{s}$ & \multicolumn{2}{l}{$20 \mathrm{~m} / \mathrm{s}$} & \multicolumn{2}{l}{$60 \mathrm{~m} / \mathrm{s}$} & $100 \mathrm{~m} / \mathrm{s}$ \\
& $\mathrm{S} / \mathrm{C}$ & $\mathrm{C} / \mathrm{C}$ & $\mathrm{S} / \mathrm{C}$ & $\mathrm{C} / \mathrm{C}$ & $\mathrm{S} / \mathrm{C}$ & $\mathrm{C} / \mathrm{C}$ & $\mathrm{S} / \mathrm{C}$ & $\mathrm{C} / \mathrm{C}$ \\
\hline $0 \mathrm{~dB}$ & 93 & 203 & 119 & 161 & 121 & 154 & 141 & 155 \\
$2 \mathrm{~dB}$ & 5 & 20 & 11 & 10 & 18 & 7 & 36 & 9 \\
$4 \mathrm{~dB}$ & 0 & 0 & 8 & 0 & 18 & 0 & 23 & 0 \\
$10 \mathrm{~dB}$ & 1 & 0 & 5 & 0 & 21 & 0 & 22 & 0 \\
$40 \mathrm{~dB}$ & 0 & 0 & 6 & 0 & 16 & 0 & 30 & 0 \\
\hline
\end{tabular}

Fuente: Armijo, 2017 
Tabla 3 BOC con y sin codificación convolucional $(2,1,3)$

\begin{tabular}{|c|c|c|c|c|c|c|c|c|}
\hline \multirow{2}{*}{ SNR } & \multicolumn{2}{|c|}{$0 \mathrm{~m} / \mathrm{s}$} & \multicolumn{2}{|c|}{$20 \mathrm{~m} / \mathrm{s}$} & \multicolumn{2}{|c|}{$60 \mathrm{~m}$} & \multirow{2}{*}{\multicolumn{2}{|c|}{$\begin{array}{l}100 \mathrm{~m} / \mathrm{s} \\
\mathrm{S} / \mathrm{C} \mathrm{C} / \mathrm{C}\end{array}$}} \\
\hline & $\mathrm{S} / \mathrm{C}$ & $\mathrm{C} / \mathrm{C}$ & $\mathrm{S} / \mathrm{C}$ & $\mathrm{C} / \mathrm{C}$ & $\mathrm{S} / \mathrm{C}$ & $\mathrm{C} / \mathrm{C}$ & & \\
\hline $0 \mathrm{~dB}$ & 81 & 191 & 114 & 156 & 116 & 149 & 133 & 147 \\
\hline $2 \mathrm{~dB}$ & 0 & 14 & 10 & 9 & 17 & 6 & 34 & 7 \\
\hline $4 \mathrm{~dB}$ & 0 & 0 & 5 & 0 & 16 & 0 & 18 & 0 \\
\hline $10 \mathrm{~dB}$ & 0 & 0 & 4 & 0 & 19 & 0 & 19 & 0 \\
\hline $40 \mathrm{~dB}$ & 0 & 0 & 5 & 0 & 14 & 0 & 25 & 0 \\
\hline
\end{tabular}

Fuente: Andrea Llerena, 2019

\section{BER en Modulación BPSK y BOC en canal de Rayleigh}

Las Tablas 4 y 5, muestran los resultados del BER obtenidos cuando la señal GPS L1, atraviesa el canal de transmisión Rayleigh, a diferentes velocidades.

Tabla 4 BER de BPSK con y sin codificación convolucional $(2,1,3)$

\begin{tabular}{|c|c|c|c|c|c|c|c|c|}
\hline \multirow{2}{*}{$\begin{array}{l}\text { Velocidad } \\
\text { SNR }\end{array}$} & \multicolumn{2}{|l|}{$0 \mathrm{~m} / \mathrm{s}$} & \multicolumn{2}{|l|}{$20 \mathrm{~m} / \mathrm{s}$} & \multicolumn{2}{|l|}{$60 \mathrm{~m} / \mathrm{s}$} & \multicolumn{2}{|c|}{$100 \mathrm{~m} / \mathrm{s}$} \\
\hline & $\mathrm{S} / \mathrm{C}$ & $\mathrm{C} / \mathrm{C}$ & $\mathrm{S} / \mathrm{C}$ & $\mathrm{C} / \mathrm{C}$ & $\mathrm{S} / \mathrm{C}$ & $\mathrm{C} / \mathrm{C}$ & $\mathrm{S} / \mathrm{C}$ & $\mathrm{C} / \mathrm{C}$ \\
\hline $0 \mathrm{Db}$ & $\begin{array}{l}2.27 \mathrm{E}- \\
03\end{array}$ & $\begin{array}{l}4.96 \mathrm{E}- \\
03\end{array}$ & $\begin{array}{l}2.91 \mathrm{E}- \\
03\end{array}$ & $\begin{array}{l}3.93 \mathrm{E}- \\
03\end{array}$ & $\begin{array}{l}2.96 \mathrm{E}- \\
03\end{array}$ & $\begin{array}{l}3.76 \mathrm{E}- \\
03\end{array}$ & $\begin{array}{l}3.45 \mathrm{E}- \\
03\end{array}$ & $\begin{array}{l}3.79 \mathrm{E}- \\
03\end{array}$ \\
\hline $2 \mathrm{~dB}$ & $\begin{array}{l}1.22 \mathrm{E}- \\
04\end{array}$ & $\begin{array}{l}4.89 \mathrm{E}- \\
04\end{array}$ & $\begin{array}{l}2.69 \mathrm{E}- \\
04\end{array}$ & $\begin{array}{l}2.44 \mathrm{E}- \\
04\end{array}$ & $\begin{array}{l}4.40 \mathrm{E}- \\
04\end{array}$ & $\begin{array}{l}1.71 \mathrm{E}- \\
04\end{array}$ & $\begin{array}{l}8.80 \mathrm{E}- \\
04\end{array}$ & $\begin{array}{l}2.20 \mathrm{E}- \\
04\end{array}$ \\
\hline $4 \mathrm{~dB}$ & 0 & 0 & $\begin{array}{l}1.96 \mathrm{E}- \\
04\end{array}$ & 0 & $\begin{array}{l}4.40 \mathrm{E}- \\
04\end{array}$ & 0 & $\begin{array}{l}5.62 \mathrm{E}- \\
04\end{array}$ & 0 \\
\hline $10 \mathrm{~dB}$ & $\begin{array}{l}2.44 \mathrm{E}- \\
05\end{array}$ & 0 & $\begin{array}{l}1.22 \mathrm{E}- \\
04\end{array}$ & 0 & $\begin{array}{l}5.13 \mathrm{E}- \\
04\end{array}$ & 0 & $\begin{array}{l}5.38 \mathrm{E}- \\
04\end{array}$ & 0 \\
\hline $40 \mathrm{~dB}$ & 0 & 0 & $\begin{array}{l}1.47 \mathrm{E}- \\
04\end{array}$ & 0 & $\begin{array}{l}3.91 \mathrm{E}- \\
04\end{array}$ & 0 & $\begin{array}{l}7.33 \mathrm{E}- \\
04\end{array}$ & 0 \\
\hline
\end{tabular}

Fuente: Armijo, 2017

Tabla 5 BER de BOC con y sin codificación convolucional $(2,1,3)$

\begin{tabular}{lllllllll}
\hline Velocidad & $0 \mathrm{~m} / \mathrm{s}$ & & $20 \mathrm{~m} / \mathrm{s}$ & & $60 \mathrm{~m} / \mathrm{s}$ & \multicolumn{3}{c}{$100 \mathrm{~m} / \mathrm{s}$} \\
$\mathrm{SNR}$ & $\mathrm{S} / \mathrm{C}$ & $\mathrm{C} / \mathrm{C}$ & $\mathrm{S} / \mathrm{C}$ & $\mathrm{C} / \mathrm{C}$ & $\mathrm{S} / \mathrm{C}$ & $\mathrm{C} / \mathrm{C}$ & $\mathrm{S} / \mathrm{C}$ & $\mathrm{C} / \mathrm{C}$ \\
\hline \multirow{2}{*}{$0 \mathrm{~dB}$} & $1.98 \mathrm{E}-$ & $4.67 \mathrm{E}-$ & $2.79 \mathrm{E}-$ & $3.81 \mathrm{E}-$ & $2.83 \mathrm{E}-$ & $3.64 \mathrm{E}-$ & $3.25 \mathrm{E}-$ & $3.59 \mathrm{E}-$ \\
& 03 & 03 & 03 & 03 & 03 & 03 & 03 & 03 \\
$2 \mathrm{~dB}$ & 0 & $3.42 \mathrm{E}-$ & $2.44 \mathrm{E}-$ & $2.20 \mathrm{E}-$ & $4.15 \mathrm{E}-$ & $1.47 \mathrm{E}-$ & $8.31 \mathrm{E}-$ & $1.71 \mathrm{E}-$ \\
& & 04 & 04 & 04 & 04 & 04 & 04 & 04 \\
$4 \mathrm{~dB}$ & 0 & 0 & $1.22 \mathrm{E}-$ & 0 & $3.91 \mathrm{E}-$ & & $4.40 \mathrm{E}-$ & 0 \\
& & & 04 & 0 & 04 & 0 & 04 & 0
\end{tabular}




\begin{tabular}{|c|c|c|c|c|c|c|c|}
\hline $10 \mathrm{~dB}$ & 0 & 0 & $\begin{array}{l}9.78 \mathrm{E}- \\
05\end{array}$ & 0 & $\begin{array}{l}4.64 \mathrm{E}- \\
04\end{array}$ & 0 & $\begin{array}{l}4.64 \mathrm{E}- \\
04\end{array}$ \\
\hline $40 \mathrm{~dB}$ & 0 & 0 & $\begin{array}{l}1.22 \mathrm{E}- \\
04\end{array}$ & 0 & $\begin{array}{l}3.42 \mathrm{E}- \\
04\end{array}$ & 0 & $\begin{array}{l}6.11 \mathrm{E}- \\
04\end{array}$ \\
\hline
\end{tabular}

Fuente: Andrea Llerena, 2019

\section{Evaluación de la Modulación BPSK y BOC en canal de Rician}

En las Tablas 6 y 7, se muestran los errores producidos dentro del canal con desvanecimiento tipo Rician sin y con codificación Convolucional $(2,1,3)$; a los que se les aplicaron las modulaciones BPSK y BOC, respectivamente.

Tabla 6 BPSK con y sin codificación convolucional $(2,1,3)$

\begin{tabular}{llllllllll}
\hline SNR & $\mathbf{0 ~ m} / \mathbf{s}$ & \multicolumn{2}{l}{$\mathbf{2 0} \mathbf{~ m} / \mathbf{s}$} & $\mathbf{6 0} \mathbf{~ m} / \mathbf{s}$ & \multicolumn{2}{l}{$\mathbf{1 0 0} \mathbf{~ m} / \mathbf{s}$} \\
& S/C & C/C & S/C & C/C & S/C & C/C & S/C & C/C \\
\hline $0 \mathrm{~dB}$ & 140 & 165 & 126 & 196 & 98 & 150 & 122 & 159 \\
$2 \mathrm{~dB}$ & 10 & 14 & 20 & 7 & 16 & 18 & 24 & 9 \\
$4 \mathrm{~dB}$ & 1 & 0 & 4 & 0 & 8 & 0 & 6 & 0 \\
$10 \mathrm{~dB}$ & 0 & 0 & 4 & 0 & 4 & 0 & 20 & 0 \\
$40 \mathrm{~dB}$ & 0 & 0 & 0 & 0 & 4 & 0 & 12 & 0 \\
\hline
\end{tabular}

Fuente: Armijo, 2017

Tabla 7 BOC con y sin codificación convolucional $(2,1,3)$

\begin{tabular}{llllllllll}
\hline $\mathrm{SNR}$ & $0 \mathrm{~m} / \mathrm{s}$ & \multicolumn{2}{l}{$20 \mathrm{~m} / \mathrm{s}$} & \multicolumn{2}{l}{$60 \mathrm{~m} / \mathrm{s}$} & $100 \mathrm{~m} / \mathrm{s}$ \\
& $\mathrm{S} / \mathrm{C}$ & $\mathrm{C} / \mathrm{C}$ & $\mathrm{S} / \mathrm{C}$ & $\mathrm{C} / \mathrm{C}$ & $\mathrm{S} / \mathrm{C}$ & $\mathrm{C} / \mathrm{C}$ & $\mathrm{S} / \mathrm{C}$ & $\mathrm{C} / \mathrm{C}$ \\
\hline $0 \mathrm{~dB}$ & 132 & 157 & 101 & 171 & 95 & 147 & 113 & 150 \\
$2 \mathrm{~dB}$ & 8 & 12 & 18 & 6 & 11 & 13 & 22 & 8 \\
$4 \mathrm{~dB}$ & 0 & 0 & 1 & 0 & 5 & 0 & 3 & 0 \\
$10 \mathrm{~dB}$ & 0 & 0 & 1 & 0 & 4 & 0 & 15 & 0 \\
$40 \mathrm{~dB}$ & 0 & 0 & 0 & 0 & 1 & 0 & 7 & 0 \\
\hline
\end{tabular}

Fuente: Andrea Llerena, 2019

\section{BER en Modulación BPSK y BOC en canal de Rician}

Las Tablas 8 y 9 muestran los resultados obtenidos cuando la señal GPS L1, atraviesa el canal de transmisión Rician, a diferentes velocidades. 
Tabla 8BER de BPSK con y sin codificación convolucional $(2,1,3)$

\begin{tabular}{lllllllll}
\hline $\mathrm{SNR}$ & $0 \mathrm{~m} / \mathrm{s}$ & \multicolumn{3}{c}{$20 \mathrm{~m} / \mathrm{s}$} & \multicolumn{2}{l}{$60 \mathrm{~m} / \mathrm{s}$} & \multicolumn{2}{l}{$100 \mathrm{~m} / \mathrm{s}$} \\
& $\mathrm{S} / \mathrm{C}$ & $\mathrm{C} / \mathrm{C}$ & $\mathrm{S} / \mathrm{C}$ & $\mathrm{C} / \mathrm{C}$ & $\mathrm{S} / \mathrm{C}$ & $\mathrm{C} / \mathrm{C}$ & $\mathrm{S} / \mathrm{C}$ & $\mathrm{C} / \mathrm{C}$ \\
\hline $0 \mathrm{~dB}$ & $3.42 \mathrm{E}-03$ & $4.03 \mathrm{E}-03$ & $3.08 \mathrm{E}-03$ & $4.79 \mathrm{E}-03$ & $2.39 \mathrm{E}-03$ & $3.67 \mathrm{E}-03$ & $2.98 \mathrm{E}-03$ & $3.89 \mathrm{E}-03$ \\
$2 \mathrm{~dB}$ & $2.44 \mathrm{E}-04$ & $3.42 \mathrm{E}-04$ & $4.89 \mathrm{E}-04$ & $1.71 \mathrm{E}-04$ & $3.91 \mathrm{E}-04$ & $4.40 \mathrm{E}-04$ & $5.87 \mathrm{E}-04$ & $2.20 \mathrm{E}-04$ \\
$4 \mathrm{~dB}$ & $2.44 \mathrm{E}-05$ & 0 & $9.78 \mathrm{E}-05$ & 0 & $1.96 \mathrm{E}-04$ & 0 & $1.47 \mathrm{E}-04$ & 0 \\
$10 \mathrm{~dB}$ & 0 & 0 & $9.78 \mathrm{E}-05$ & 0 & $9.78 \mathrm{E}-05$ & 0 & $4.89 \mathrm{E}-04$ & 0 \\
$40 \mathrm{~dB}$ & 0 & 0 & 0 & 0 & $9.78 \mathrm{E}-05$ & 0 & $2.93 \mathrm{E}-04$ & 0 \\
\hline
\end{tabular}

Fuente: Armijo, 2017

Tabla 9BER de BOC con y sin codificación convolucional $(2,1,3)$

\begin{tabular}{lllllllll}
\hline $\mathrm{SNR}$ & $0 \mathrm{~m} / \mathrm{s}$ & & $20 \mathrm{~m} / \mathrm{s}$ & \multicolumn{3}{c}{$60 \mathrm{~m} / \mathrm{s}$} & \multicolumn{3}{c}{$100 \mathrm{~m} / \mathrm{s}$} \\
& $\mathrm{S} / \mathrm{C}$ & $\mathrm{C} / \mathrm{C}$ & S/C & $\mathrm{C} / \mathrm{C}$ & S/C & $\mathrm{C} / \mathrm{C}$ & S/C & $\mathrm{C} / \mathrm{C}$ \\
\hline $0 \mathrm{~dB}$ & $3.23 \mathrm{E}-03$ & $3.84 \mathrm{E}-03$ & $2.47 \mathrm{E}-03$ & $4.18 \mathrm{E}-03$ & $2.32 \mathrm{E}-03$ & $3.59 \mathrm{E}-03$ & $2.76 \mathrm{E}-03$ & $3.67 \mathrm{E}-03$ \\
$2 \mathrm{~dB}$ & $1.96 \mathrm{E}-04$ & $2.93 \mathrm{E}-04$ & $4.40 \mathrm{E}-04$ & $1.47 \mathrm{E}-04$ & $2.69 \mathrm{E}-04$ & $3.18 \mathrm{E}-04$ & $5.38 \mathrm{E}-04$ & $1.96 \mathrm{E}-04$ \\
$4 \mathrm{~dB}$ & 0 & 0 & $2.44 \mathrm{E}-05$ & 0 & $1.22 \mathrm{E}-04$ & 0 & $7.33 \mathrm{E}-05$ & 0 \\
$10 \mathrm{~dB}$ & 0 & 0 & $2.44 \mathrm{E}-05$ & 0 & $9.78 \mathrm{E}-05$ & 0 & $3.67 \mathrm{E}-04$ & 0 \\
$40 \mathrm{~dB}$ & 0 & 0 & 0 & 0 & $2.44 \mathrm{E}-05$ & 0 & $1.71 \mathrm{E}-04$ & 0 \\
\hline
\end{tabular}

Fuente: Andrea Llerena, 2019

\section{RESULTADOS}

\section{Canal Rayleigh}

Las figuras 7, 8, 9 y 10 muestran la Tasa de Error Binaria (BER) vs la Relación Señal a Ruido (SNR) para una modulación BPSK vs. modulación BOC, Sin y con el uso del codificador convolucional $(2,1,3)$ en el canal Rayleigh, para las velocidades de receptor $0 \mathrm{~m} / \mathrm{s}, 20 \mathrm{~m} / \mathrm{s}, 60 \mathrm{~m} / \mathrm{s}$ y $100 \mathrm{~m} / \mathrm{s}$.

Figura 7. SNR vs. BER en un Canal Rayleigh a $0 \mathrm{~m} / \mathrm{s}$

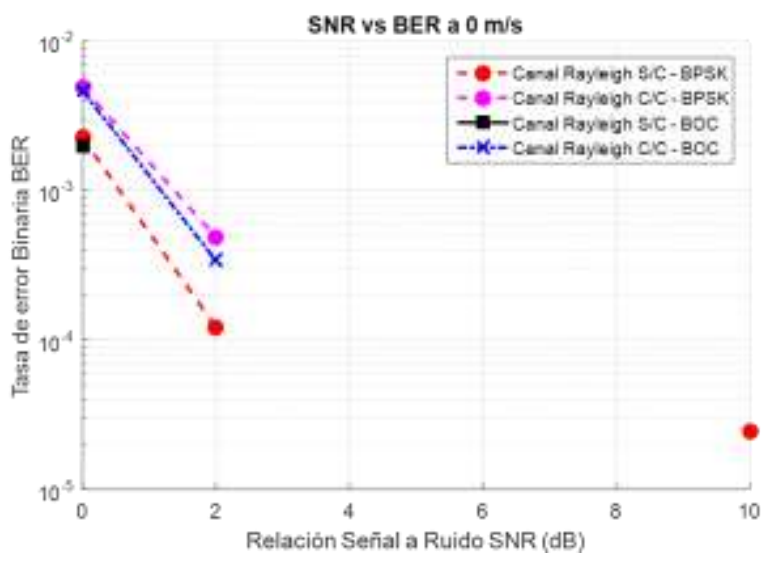


ISSN: 2602-8085

Figura 8. SNR vs. BER en un Canal Rayleigh a $20 \mathrm{~m} / \mathrm{s}$

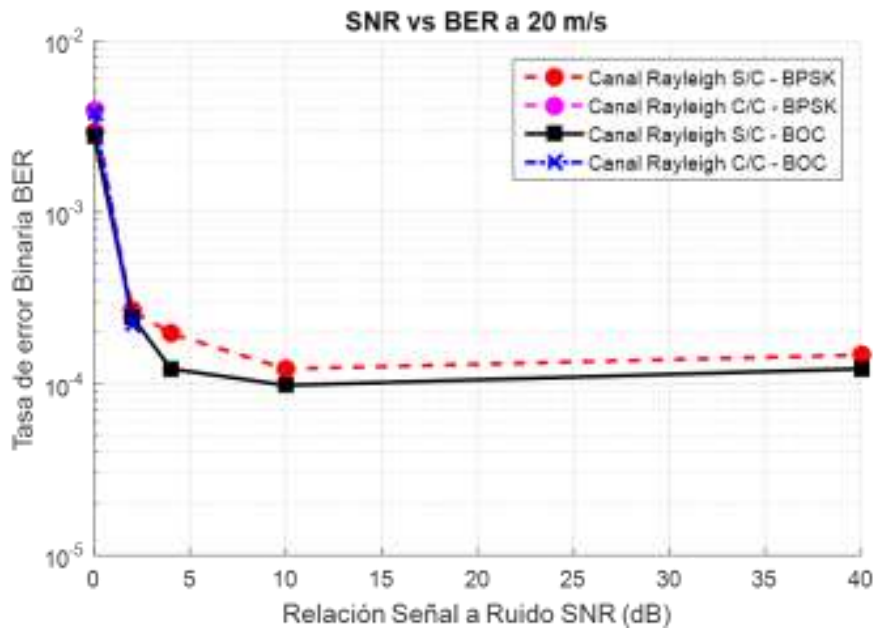

Figura 9. SNR vs. BER en un Canal Rayleigh a $60 \mathrm{~m} / \mathrm{s}$

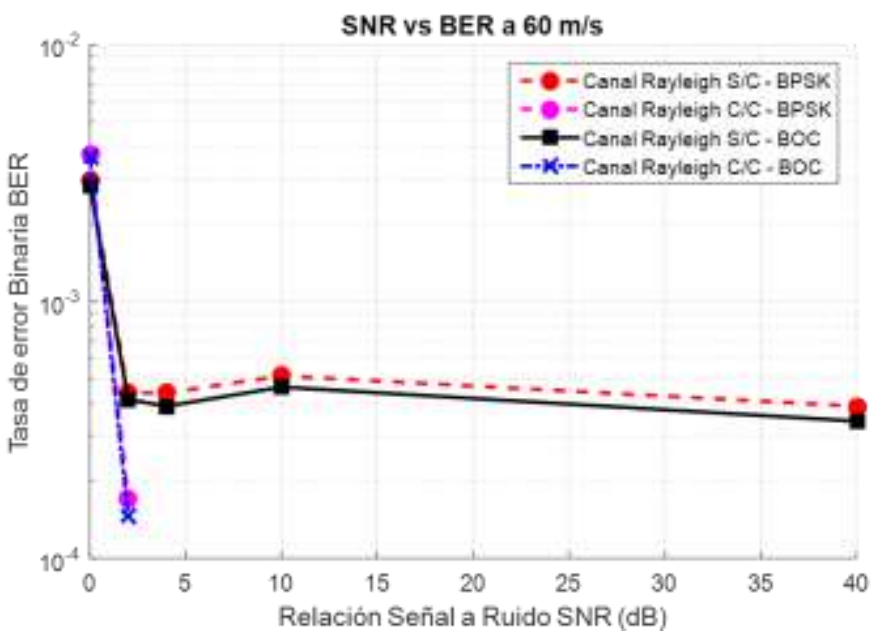

Figura 10. SNR vs. BER en un Canal Rayleigh a 100 m/s

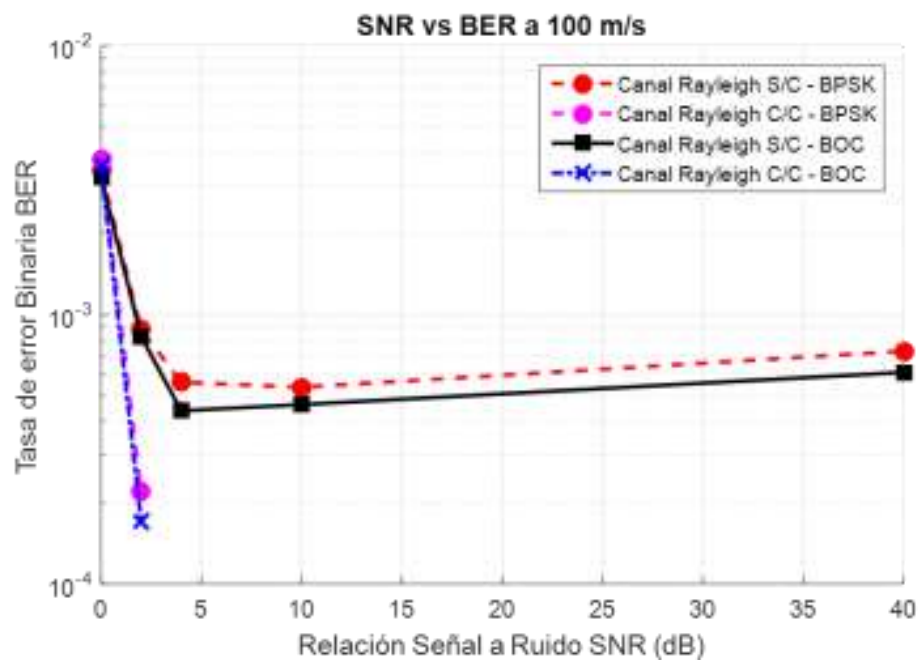


ISSN: 2602-8085

Vol. 3, N³, p. 225-241, julio-septiembre, 2019

En la figura 11, se muestra en forma tabulada la cantidad de errores presentes en el canal Rayleigh, para los dos tipos de modulación.

Figura 11. Errores generados en el canal Rayleigh con Modulación BPSK y BOC

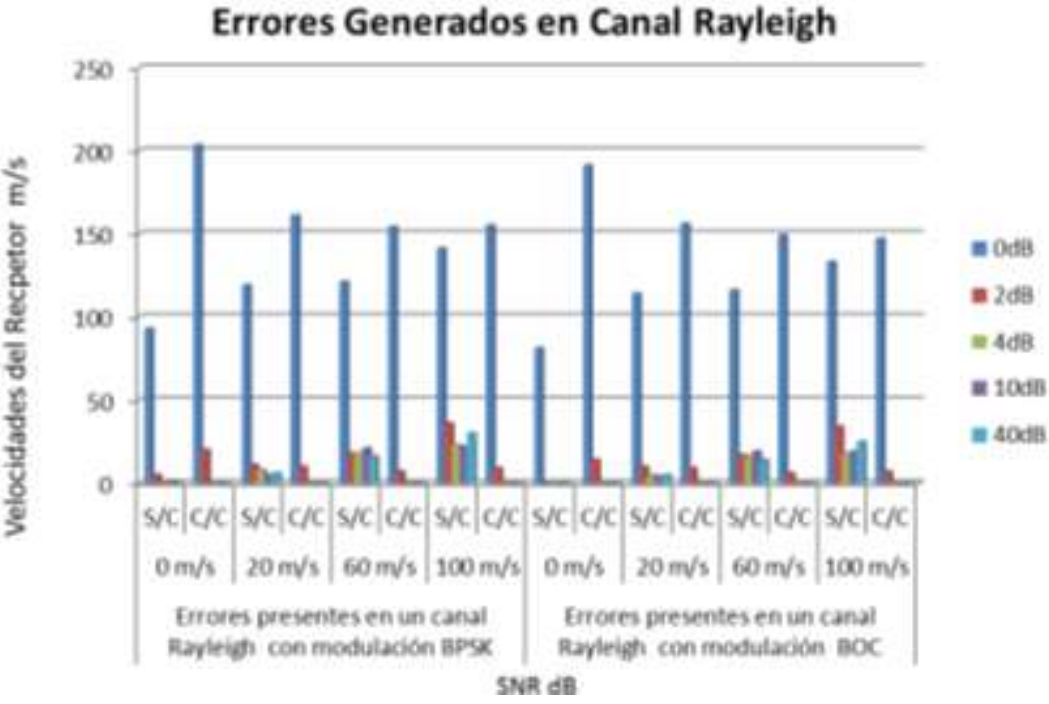

\section{Canal Rician}

Figura 12. SNR vs. BER en un Canal Rician a $0 \mathrm{~m} / \mathrm{s}$

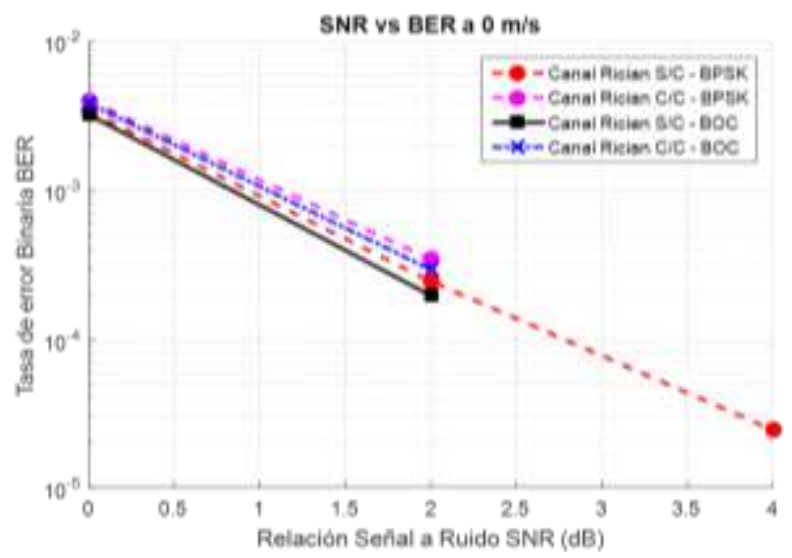

Figura 13. SNR vs. BER en un Canal Rician a $20 \mathrm{~m} / \mathrm{s}$

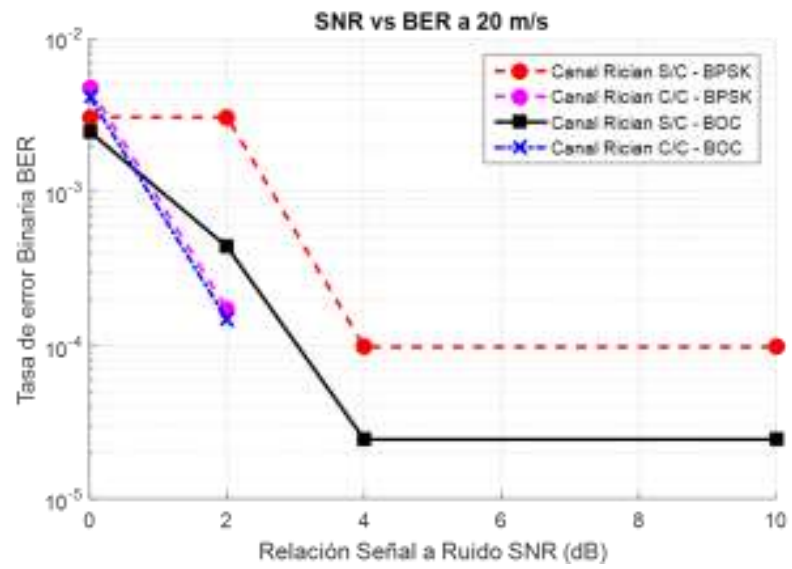


Figura 14. SNR vs. BER en un Canal Rician a $60 \mathrm{~m} / \mathrm{s}$

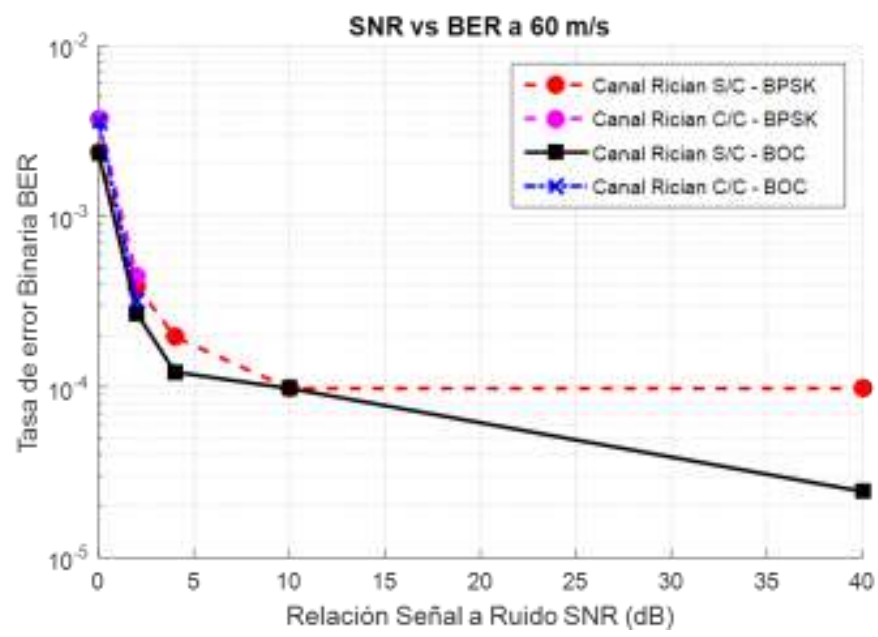

Figura 15. SNR vs. BER en un Canal Rician a 100 m/s

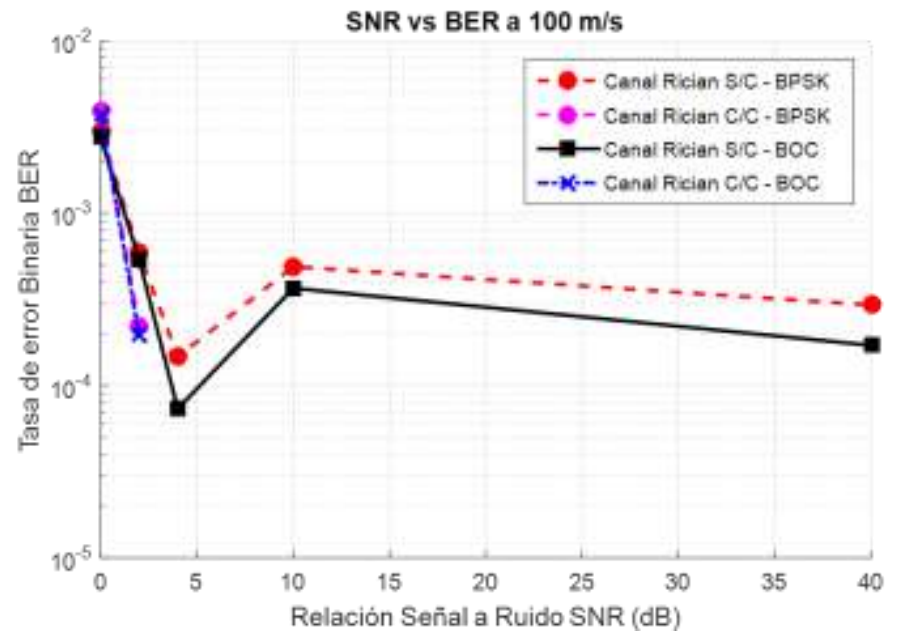

La figura 16, se muestra en forma tabulada la cantidad de errores presentes en el canal Rician, para los dos tipos de modulación.

Figura 26. Errores generados en el Canal Rician

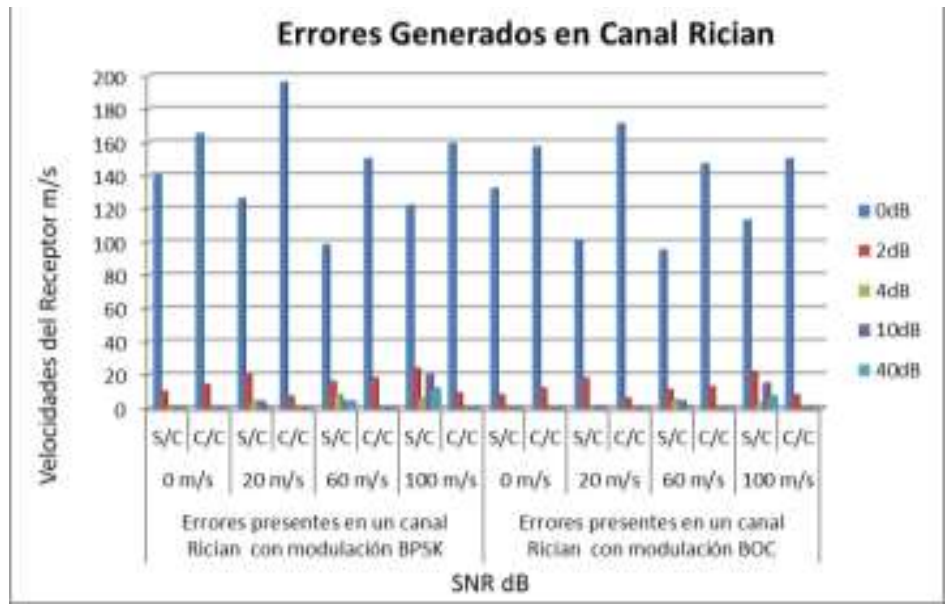




\section{Conclusiones}

- La revisión del estado de arte ha permitido dar cuenta que el desarrollo de los sistemas de posicionamiento GNSS no se detiene, por tanto requieren mejoras en la precisión y confiabilidad de la posición, lo que ofrece este tipo de modulación Binary Offset Carrier.

- La técnica de modulación Binary Offset Carrier BOC, separa el espectro de frecuencia de la señal GPS civil en dos lóbulos principales simétricos con ancho de banda de $2 \mathrm{MHz}$, debido a la presencia de la función de autocorrelación de múltiples picos que divide la densidad espectral de potencia lo que proporciona una mayor capacidad de recuperación de bits.

- Mediante la codificación Convolucional $(2,1,3)$ se pudo detectar y corregir errores de transmisión en forma eficaz a partir de los 4 decibeles de relación señal a ruido. Antes de 4 decibeles se obtuvieron mayor cantidad de bits erróneos, esto debido que el ruido es mayor que la relación SNR, en estos casos la codificación Convolucional en lugar de corregir empeora la recepción de los datos.

- Para una probabilidad de error de 10-3 para el esquema de modulación BOC en un canal Rayleigh con codificación convolucional $(2,1,3)$ a una velocidad de recepción de $100 \mathrm{~m} / \mathrm{s}$ la relación señal a ruido SNR corresponde a $0.81 \mathrm{~dB}$, que es menor en $0.015 \mathrm{~dB}$ con relación al esquema de modulación BPSK. Por lo tanto, se comprueba que la modulación BOC es más robusta frente a la presencia de ruido lo que permite obtener una menor cantidad de bits erróneos.

- Para una probabilidad de error de 10-3 para el esquema de modulación BOC en un canal Rician con codificación convolucional $(2,1,3)$ a una velocidad de $100 \mathrm{~m} / \mathrm{s}$ la relación señal a ruido SNR corresponde a $0.84 \mathrm{~dB}$, que es menor en $0.12 \mathrm{~dB}$ con relación al esquema de modulación BPSK. Permitiendo determinar que la modulación BPSK es menos robusta frente a la presencia de ruido y la modulación BOC es más resistente al ruido y la interferencia, y por tanto presenta menor cantidad de bits errados.

\section{Referencias Bibliográficas}

Armijo, I. (2017). Desarrollo de un algoritmo de la técnica de espectro ensanchado en Matlab para evaluar el rendimiento del canal de transmisión en GPS L1. Tesis de Ingeniería. Riobamba, Ecuador.

Betz, J. W. (25-27 de Enero de 1999). The Offset Carrier Modulation for GPS Modernization. 639-648. The Institute of Navigation, San Diego, California.

Hofmann-Wellenhof, B., Lichtenegger, H., \& Wasle, E. (2008). "GNSS - Global Navigation Satellite Systems GPS, GLONASS, Galileo and more”. Austria: Springer Wien NewYork.

Julien, O. (2014). Future GNSS Signals. ENAC - Ecole Nationale de L'Aviation Civile. Francia. 
Reyes, J. (2012). Detección de señales del Sistema de Posicionamiento Global (GPS) usando MATLAB. Trabajo de Diplomado. Santa Clara, Cuba.

Serna, M. (2015). Simulación del Enlace Descendiente de Galileo. Trabajo de Fin de Grado. Léganes, Madrid.

Usha, G., Surya-Prabha, C., \& Dr.V.B.S.Srilatha-Indira, D. (2012). Simulation and Analysis of Binary Offset Carrier Modulation for Modernized Global Navigation Satellite Systems. International Journal of Engineering Research and Applications (IJERA), 2298-2304

Valadez, D. (2015). Estado de GNSS - Parte 1: Modernización de GPS. Obtenido de https://www.effigis.com/es/estado-de-gnss-parte-1-modernizacion-de-gps/

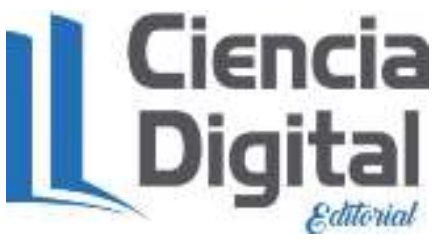




\section{PARA CITAR EL ARTÍCULO INDEXADO.}

Llerena Pintag, A., Zabala Haro, M., Pacheco Cunduri, M., Casignia Vasconez, B., \& Oñate Andino, M. (2019). Evaluación del rendimiento de la modulación Binary Offset Carrier (BOC) versus la señal Civil GPS/L1. Ciencia Digital, 3(3), 225-241. https://doi.org/10.33262/cienciadigital.v3i3.626

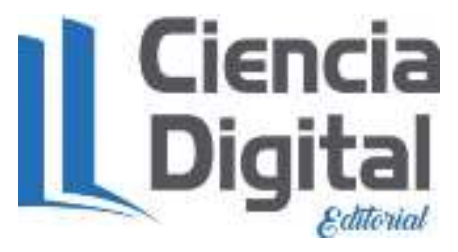

El artículo que se publica es de exclusiva responsabilidad de los autores y no necesariamente reflejan el pensamiento de la Revista Ciencia Digital.

El artículo queda en propiedad de la revista y, por tanto, su publicación parcial y/o total en otro medio tiene que ser autorizado por el director de la Revista Ciencia Digital.
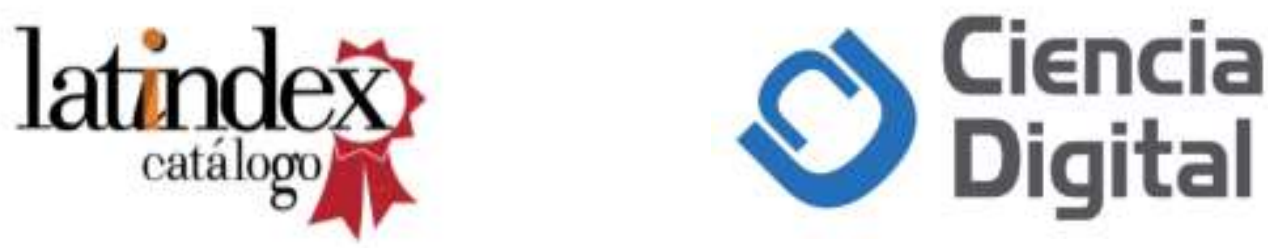